\title{
DEMONSTRATION OF A NEW APPROACH FOR MEASURING TOOLS WITH THE IMPINGEMENT SOUND OF AN AIR JET USING MACHINE LEARNING
}

\author{
H. Wuerschinger ${ }^{1 *}$, D. Gross ${ }^{1}$, M. Muehlbauer ${ }^{1}$, M. Stadler ${ }^{1}$, N. Hanenkamp ${ }^{1}$ \\ ${ }^{1}$ University Erlangen-Nuremberg, Institute of Resource and Energy Efficient Production Systems, Fürth, Germany \\ ${ }^{*}$ Corresponding author; e-mail: hubert.wuerschinger@fau.de
}

\begin{abstract}
Monitoring the tool condition of machining processes is important but challenging. Several automated tool condition monitoring solutions are available, but often not used due to existing restrictions or disadvantages. A new approach can be the detection and measurement of tool conditions analyzing the sound of an air jet impingement on tools. Due to the availability of compressed air as a working and cleaning medium for many processes, this approach can be used for various condition monitoring and measuring tasks. In this paper the procedure and its functionality are first presented on simple shapes and then tested on the tool wear of inserts.
\end{abstract}

\section{Keywords:}

Tool Condition Monitoring; Condition Monitoring; Impingement Sound; Jet Impingement; Air Jet; Flow Noise; Airborne Sound; Machine Learning

\section{INTRODUCTION}

The evaluation of the performance of a machining process depends on the quality of the workpieces [Rehorn 2005] and the productivity [Liu 2019]. The cutting tool as the link between machine and the workpiece has a major influence on the process performance. In this context, tool wear plays a significant role. From the quality perspective, the tool state influences the workpiece quality. From the economical perspective, it is estimated that up to $20 \%$ of the machine downtimes are attributed to failures of the cutting tools [Vetrichelvan 2015], [Bhattacharyya 2007]. As a result, the tools are changed early, often before the actual end of their life is reached. This leads to potential savings that can be achieved by using the full capacity of the tool life. It is estimated that costs of the tools and their changes account for $3-12 \%$ of the processing costs [Zhou 2018].

All these aspects describe the need to monitor the condition of the cutting tools and replace them in time before failures occur and also try to use them as long as possible to reduce the tool replacement costs [Aliustaoglu 2009]. In practice, several approaches are common for Tool Condition Monitoring (TCM) with different advantages and disadvantages.

In this paper, a new approach for TCM with a wide range of applications is introduced. The measurement is based on the evaluation of an impingement sound that occurs when an air jet hits a tool. At the beginning of this paper, the state of the art is presented with the relevant work according to the new approach. In the practical part of this work, at first, simple geometric objects have been tested to examine its functionality. After that, the potentials to measure the tool wear is depicted by tests on cutting inserts. In the closing, the results are discussed and further research is described.

\section{STATE OF THE ART}

TCM is a method to monitor the tool state to reduce production losses. The states can be classified into tool wear, breakage, and chipping. A TCM system consists of two parts, the software and the hardware. Both are necessary to build a TCM application and to integrate it into the machine tool [Siddhpura 2013]. Sensors are used to collect information about the wear condition of the tool and pass this information on in the form of data so that decisions can be made [Kuntoğlu 2020]. In the literature, TCM systems can be categorized as direct and indirect methods as well as in continuous (online) and intermittent (offline) [Dimla 2000], [Siddhpura 2013], [Ostasevicius 2020]. The definition of direct and indirect methods in the field of TCM differs from the field of general measurement technology. In the literature of measurement technology, direct methods are described as methods in which the measured value is obtained by direct comparison with a reference value of the same measurand [Puente León 2019]. Indirect methods are described as methods in which the measured quantity is traced back to other measurands via physical relationships and determined from these [Puente 2019]. In the following, the division of TCM systems is briefly explained using the more appropriate terms online and offline. 
With offline methods it is not possible to detect the wear when the tool is in cutting mode [Dimla 2000], [Liu 2019], [Siddhpura 2013]. These methods are based on direct sensing to the tools and can be done e.g. with optical laser beams, tactile devices, proximity sensors or the workpiece electrical resistance. The offline methods have advantages in the accuracy of measuring, but environmental conditions such as chips and cutting fluids can influence the measurement process negatively.

Online measurement has its benefits in taking the measurement while the tool is in contact with the workpiece. There is no machine interruption during the measurement. The desired measurand of the tool state is traced back to physical relationships or a variable that correlates to it. This method uses factors that change depending on the state of the tool. Examples for these factors are cutting force, acoustic emission (AE), airborne sound, temperature, displacement, spindle power, torque or strain [Dimla 2000] The challenge of this method is the individual calibration for each cutting process and process adaptation. This is because the process as well as their settings such as speed, feed rate and depth of cut influence the sensor signals. Often, the measurement accuracy of offline methods is higher than that of online methods [Siddhpura 2013], [Silva 1998]. Their advantage is the usage of existing sensor signals. Also new and machine-independent sensors, or Internet of Things (IoT) Devices can be implemented to measure various signals [Ostasevicius 2020]. Especially cutting force and $A E$ signals are appropriate to measure tool wear indirectly. Table 1 depicts a qualitative comparison between offline and online methods of TCM. Commonly, the operator is responsible to monitor and determine the final tool state based on visual impressions, the acoustic noise of the process and the application of gauges. These types of diagnostic rely on the experience of the operator and is highly subjective.

Tab. 1: Comparison between the TCM methods according to [Bhuiyan 2014] and [Siddhpura 2013]

\begin{tabular}{|c|c|c|}
\hline & offline & online \\
\hline accuracy & + & - \\
\hline robustness & - & + \\
\hline $\begin{array}{l}\text { machine } \\
\text { interruption }\end{array}$ & - & + \\
\hline calibration effort & + & - \\
\hline
\end{tabular}

The new measurement method is based on the evaluation of an impingement sound which occurs when an actively generated air jet is directed towards an object. It is known that the characteristics of a flow field are changing depending on the surface. For example Nguyen et al. describe the effects of nozzle pressure ratio and nozzle-toplate distance to flow field characteristics of an underexpanded jet impinging on a flat surface [Nguyen 2019]. Inspired by bats Hwang et al. reconstructed the shape of the simple objects using machine learning $(\mathrm{ML})$ and ultrasonic sound signals which are actively sent [Hwang 2019].

In the field of manufacturing and machining, the currently known works describe the process of using airborne sound signals that occur during the operation of the machines and processes themselves [Alzahrani 2018], [Vetrichelvan 2015], [Mühlbauer 2020]. Using the resulting sound of an air jet in the field of manufacturing, Kamnis et al. introduces an approach for monitoring a thermal spray coating process with $\mathrm{ML}$ techniques to predict the coating result [Kamnis 2019].

All these approaches depict the potentials and encourage the usage of airborne sound for industrial monitoring tasks. However, none of these approaches uses an actively generated air jet and its impingement sound on objects for dimensional measurement in the field of manufacturing. Referring to the definition of measurement methods the new approach can be classified in the offline method. A benefit is that measurement and cleaning from chips and lubricant can be done in a single step. The difference to known offline methods can be seen in the combination of an offline with an online method in one system where the process calibration is done automatically. With the usage of a microphone as part of this system, the whole machining process could be monitored online.

\section{APPROACH}

The target of this paper is to introduce the functionality and the potentials of a new measuring principle for the application of TCM. The principle is based on the impingement sound when an air jet is directed towards an object. Different shapes of an object are reflected in different characteristics of the sound signals. A ML model has been used to apply a prediction of the measurand based on the features in the signals of the impingement sound. The development is a data-driven procedure. In the following, the new approach is presented starting from the setup, the data acquisition and its pre-processing, the audio signal processing, the feature selection and the determination of the height and width using ML techniques. By measuring the height and width of a tool the wear can be described. Finally, the test of this application on tool wear has been done. Figure 1 shows the approach based on the procedure of a typical ML project.

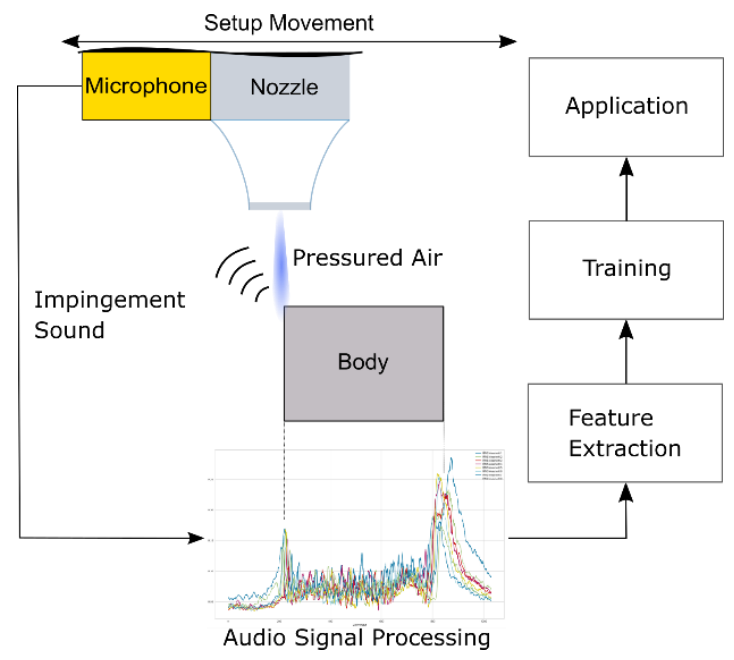

Fig. 1: Overview of the approach

The technique ML has been used because of the expected dimensionality of the feature space with its challenge to determine the function between the input factors and the output, i. e. the complex measurands.

\subsection{Experimental Setup}

For the new approach, primarily two parts are necessary which are a microphone and a nozzle. A Renkforce UM-80 conference microphone with an omnidirectional characteristic and a frequency range from 0 to $20 \mathrm{kHz}$ has been used. Three different nozzles have been tested (Tab. 2). The setup for the tests has been implemented in a Doosan DNM 500 machining centre (Fig. 2). For the proof 
of concept of the new approach an object with simple shapes, the so-called reference object (Fig. 3), has been created. With this object, a variety of data for different grades has been generated for determining the height and the width. According to TCM height and width are a simplification to describe the wear. The object has one side with eight steps. The gradation of the steps is $0.5 \mathrm{~mm}$ while the other sides are even. The aim is to identify patterns in the signals of the recorded airflow impingement sound which are correlating with the height and width using ML.

\section{Tab. 2: Overview of the tested parameters}

\begin{tabular}{|c|c|}
\hline factor & setting \\
\hline $\begin{array}{l}\text { air } \\
\text { pressure }\end{array}$ & 3 bar; 7 bar \\
\hline \multirow{4}{*}{$\begin{array}{l}\text { nozzle } \\
\text { diameter } \\
\text { and type }\end{array}$} & round flow: bore dia. $0.2 \mathrm{~mm}$ \\
\hline & \\
\hline & round flow: bore dia. $0.5 \mathrm{~mm}$ \\
\hline & flat flow: bore dia. $0.5 \mathrm{~mm}$, angle $30^{\circ}$ \\
\hline $\begin{array}{l}\text { nozzle } \\
\text { distance }\end{array}$ & $2.5 \mathrm{~mm} ; 5.0 \mathrm{~mm}$ \\
\hline feed rate & $500 \mathrm{~mm} / \mathrm{min} ; 1,500 \mathrm{~mm} / \mathrm{min}$ \\
\hline
\end{tabular}

Pre-tests with different positions of the microphone in the machining centre have been done. The best position has been found in vertically alignment with the nozzle (Fig. 2).

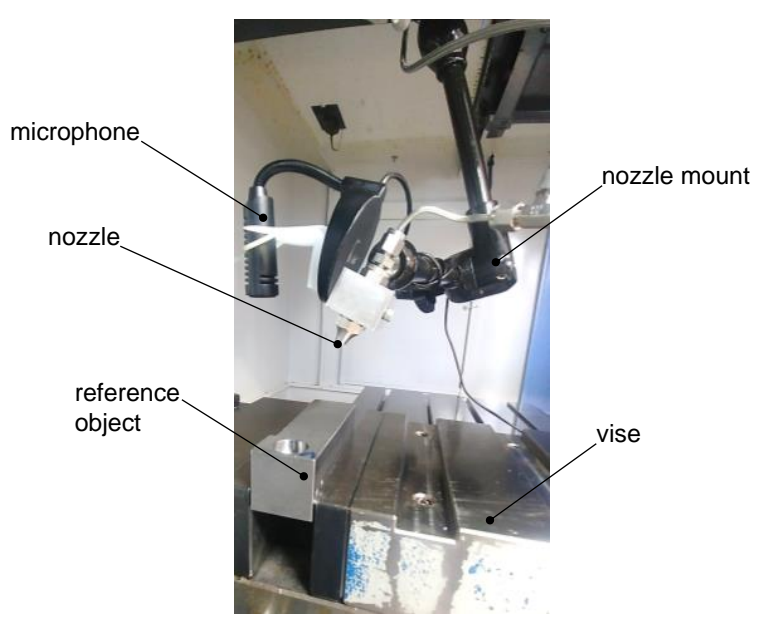

Fig. 2: Process setup in the machining centre

With this setup, the signals have been high amplitudes without oversteering and the sound signal is constant during the nozzle movement.

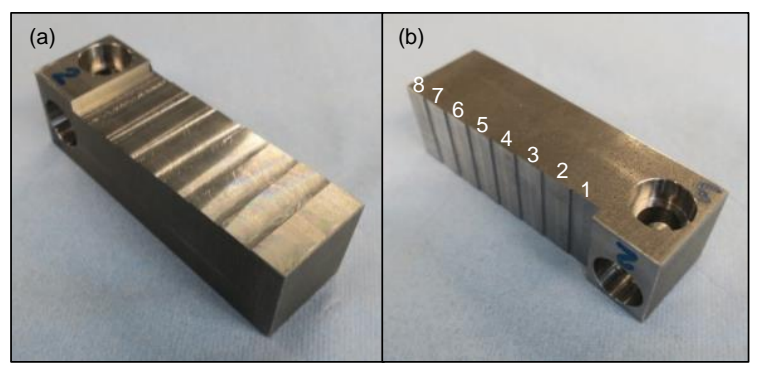

Fig. 3: Reference object with the shapes to determine: (a) height, (b) width
To ensure that the relevant element of the measured object can be found, the nozzle and thus the airflow has been moved across the measured object. This procedure can be imagined as a scanning process to find the relevant points of an object which are necessary for the measuring task. In the case of tool wear, the maximum wear has to be passed by the airflow for the diagnosis.

To find the best hardware settings experiments have been created and the data for the ML procedure has been gathered. As input factors variations of air pressure, nozzle diameter, nozzle distance and nozzle movement have been tested. The experimental variations are depicted in table 2. With the pressure at $7 \mathrm{bar}$, the round flow nozzle of $0.2 \mathrm{~mm}$ and the nozzle distance of $5.0 \mathrm{~mm}$, the loudest flow noise and impingement sound of the air jet has been created. The feed rate of $500 \mathrm{~mm} / \mathrm{min}$ results in more measuring points than in the case with a feed rate of $1,500 \mathrm{~mm} / \mathrm{min}$. The bold settings in table 2 have been used in the following investigations. For the following next steps the software Python has been used with several libraries. To generate and process the audio files the Python libraries Librosa, SciPy, NumPy and Pandas has been applied. The plots have been created with Matplotlib. For the machine learning Scikit-learn has been used.

\subsection{Data Generation and Pre-Processing}

The data generation has been carried out in the machining centre to move the nozzles relative to the reference object. The compressed air flow noise and its impingement sound on the object have been recorded by the microphone using a sampling rate of $44,100 \mathrm{~Hz}$. For further signal processing, the sound signals has been saved in a wave format file. Each file includes all eight steps of a reference object. The experiments have been repeated eight times. As a result of the manual start of the measuring process and the recording, the recorded data varies. To slice the files automatically in equal parts for every step of the reference object, a fast movement to an initial position of the machine has been applied with the NC-program. This leads to a characteristic sound pattern at the end of every file. This pattern has been used with the function cross-correlation to find in all of the recorded files at the end point of every experiment.

\subsection{Signal Processing}

To determine the dimensional features height and width of the reference object first, the sound signals have to be processed to generate spectrums from the raw audio data. In the next step, relevant features for height and width must be selected in them. Two different procedures have been used, one to determine the width and one to determine the height.

As a signal processing technique to determine the height the power spectrum density (PSD) has been generated using Welch's method which is used to compare the signals power content versus the frequencies. This method is independent of the point in time according to the impingement sound. An example of the resulting graph is shown in figure 4 . In the legend upper right, the first line (blue) from top to bottom represents the flow noise of the air jet without hitting an object. The second line (yellow) corresponds to the ambient sound of the Doosan machine. The next eight lines correspond to the impingement sound of the eight steps of the reference object for one experiment. The representations of steps 1 and 8 show anomalies in the two peaks compared to steps 2 to 7 . This could be explained by the different shapes of the steps by their outer position. For the determination of the height and width only steps 2 to 7 have been used. 


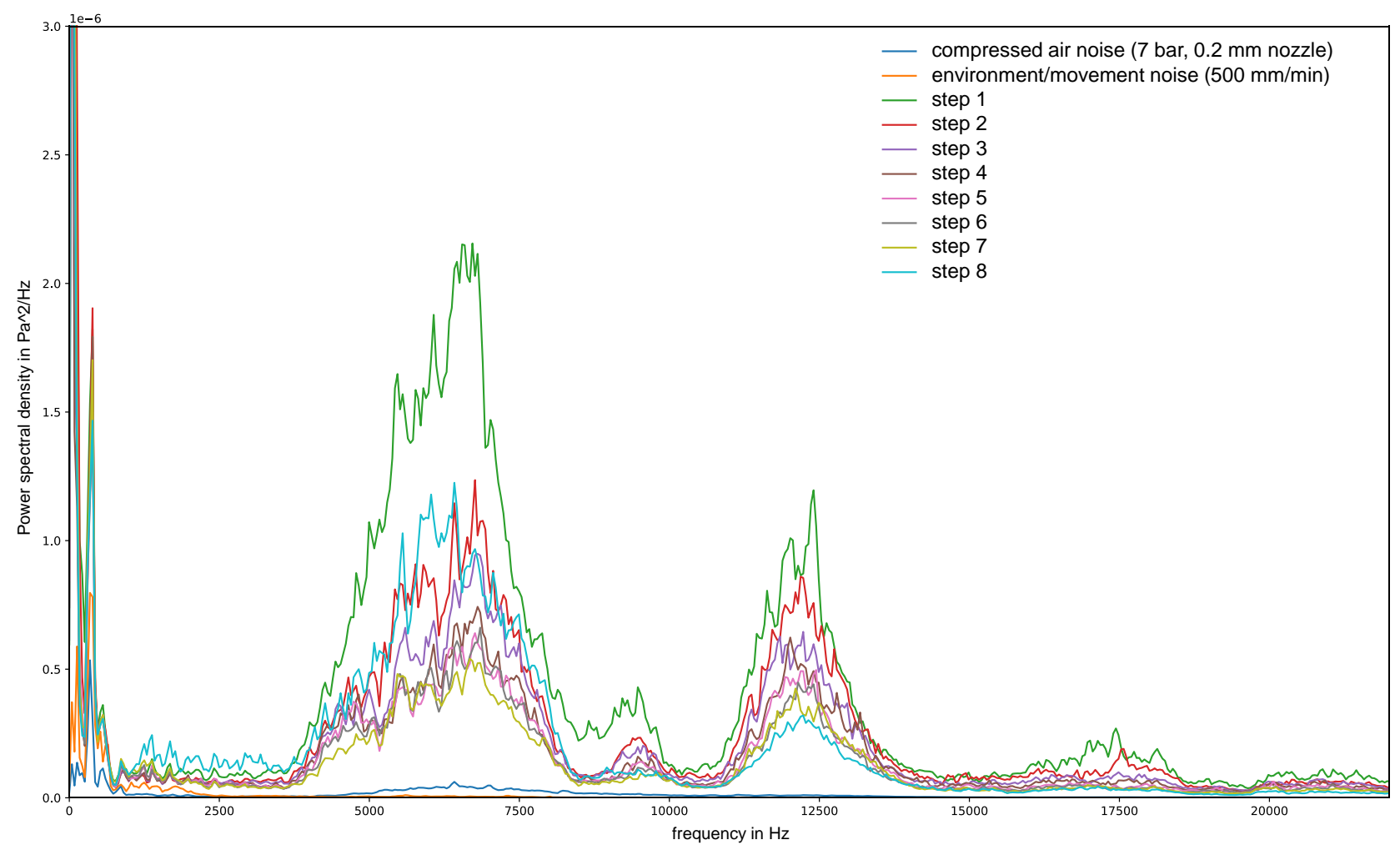

Fig. 4: Power density spectrum referring to Welch for the signal to determine the height

For application to the width of the reference object, the beginning and the end of the object must be determined. Therefore representations, i. e. their spectrum, of the frequency-domain over time have been used to find changes related to the outer dimension of the object:
- Root Mean Square (RMS)
- Spectral Centroid
- Spectral Flatness
- Spectral Bandwidth
- Spectral Roll-off
- Zero-Crossing-Rate

A scheme of the RMS of the width of the eight steps of the reference object is plotted in figure 5 for one experiment.

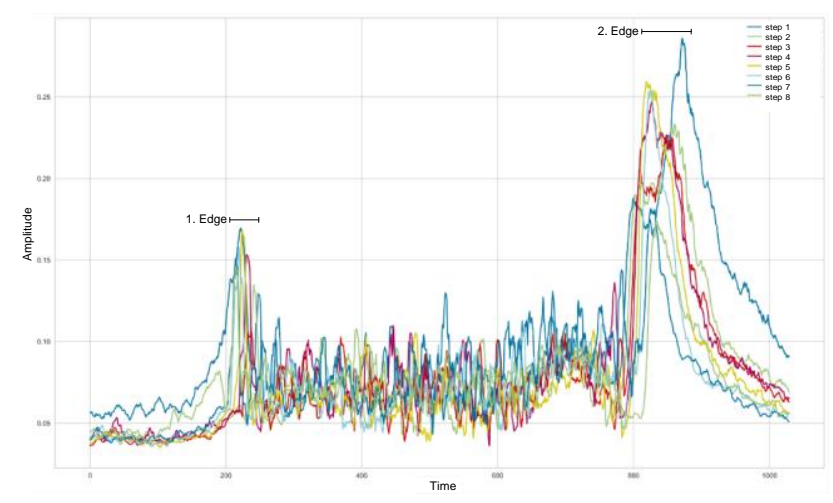

Fig. 5: RMS for the width of the eight steps of the reference object with the two peaks representing the edges

The presence of two peaks of energy can be seen for the eight steps. The left peak is representing the first edge of a step and the right peak shows the second edge of a step.
The described spectrums were used in the next sections to find features that correlate with height and width, as well as finding the best features for the ML models to build an adequate regression to measure the objects.

\subsection{Predicting the width}

In the next step, the width of the reference object has been used as the target value of a regression model. For this investigation only the six inner steps have been used, due to different characteristics of the step 1 and 8 (Fig. 5). As a dataset for the following ML procedure 48 audio files, 8 audio recordings per step, have been created with a training-to-test ratio of 80 to 20 .

For predicting the width first, the feature engineering has been applied to select features from the spectrums of the time-frequency domain referring to section 3.3. To be able to use individual values as features, the mean value (MV), the standard deviation (SD), and the difference of the maxima have been calculated from all six spectrums. The 18 resulting features have been correlated with the target value 'width' in a heatmap, shown in figure 6 . The lighter the boxes in the rightmost column, the higher the correlation between the target variable and the input variables on the left. A blue color shading indicates a negative correlation, a red color shading indicates a positive correlation. The six differences of the maxima have not being used due to their low correlation $(-0.25 \ldots+0.5)$ with the target value width. The remaining 12 features have been used for building regression models. The best model has been created with a polynomial degree of 2 using the features MV of the spectral centroid, spectral flatness and spectral bandwidth, and the SD of the RMS and spectral flatness. As a result, this model has been achieved a mean squared error (MSE) value of $0.000140 \mathrm{~mm}$ and a maximum deviation of $0.0186 \mathrm{~mm}$. Table 4 summarises the predictions on the test 


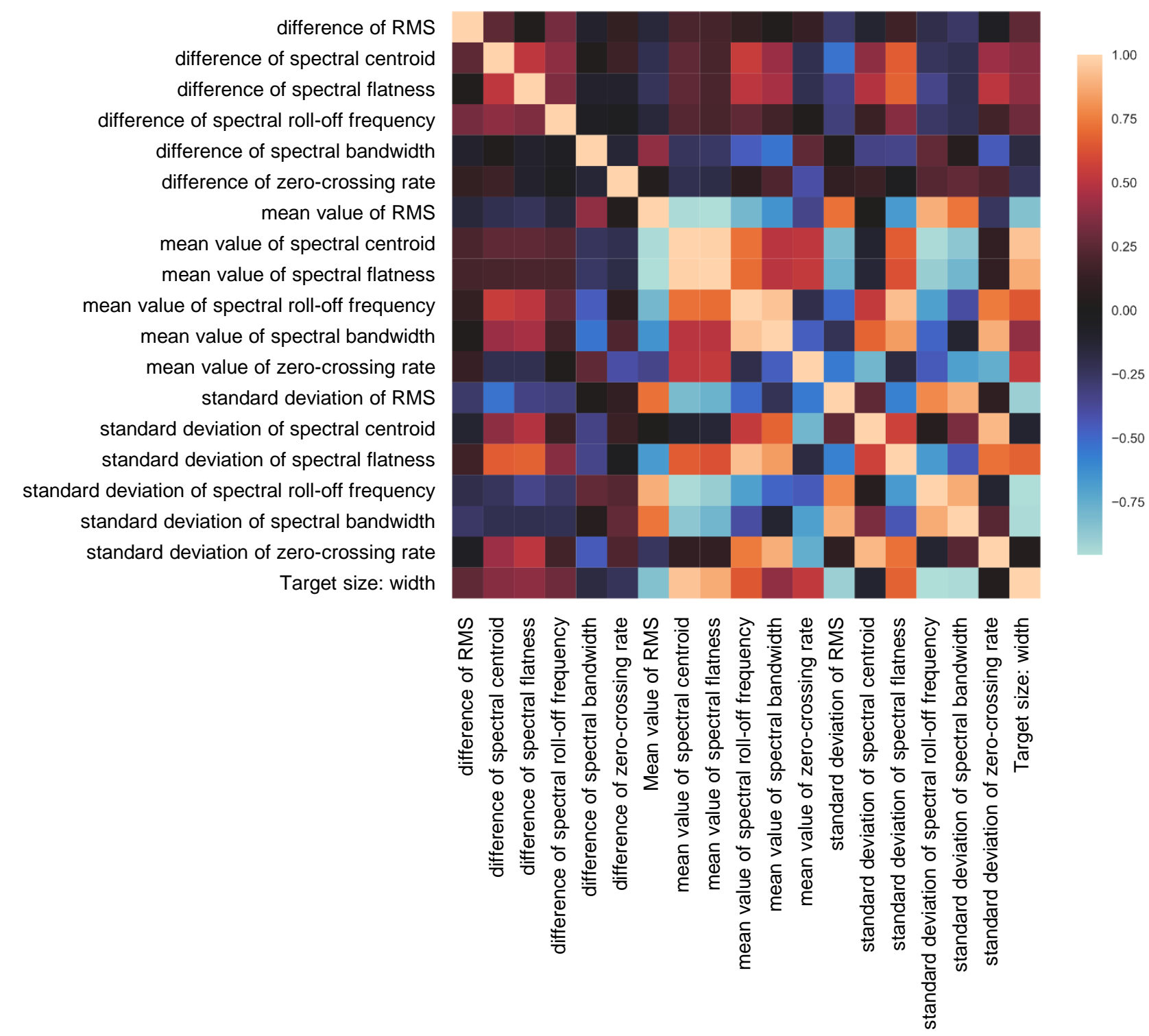

Fig. 6: Heatmap to determine the correlations for the input factors to the target value width

dataset for the best model for the six steps of the reference object and its real values.

Tab. 4: Comparison of the test data set between model predictions for width and the real values

\begin{tabular}{cccc}
\hline step & real value & $\begin{array}{c}\text { predicted } \\
\text { value }\end{array}$ & deviation \\
\hline 2 & $27.03 \mathrm{~mm}$ & $27.04226 \mathrm{~mm}$ & $0.01226 \mathrm{~mm}$ \\
3 & $27.53 \mathrm{~mm}$ & $27.53528 \mathrm{~mm}$ & $0.00528 \mathrm{~mm}$ \\
4 & $28.03 \mathrm{~mm}$ & $28.01812 \mathrm{~mm}$ & $-0.01188 \mathrm{~mm}$ \\
5 & $28.53 \mathrm{~mm}$ & $28.54860 \mathrm{~mm}$ & $0.01860 \mathrm{~mm}$ \\
6 & $29.01 \mathrm{~mm}$ & $28.99872 \mathrm{~mm}$ & $-0.01128 \mathrm{~mm}$ \\
7 & $29.49 \mathrm{~mm}$ & $29.49671 \mathrm{~mm}$ & $0.00671 \mathrm{~mm}$ \\
\hline
\end{tabular}

\subsection{Predicting the height}

The spectrum referring to Welch has been selected for predicting the height. In windows of a range of 1,000 and $2,000 \mathrm{~Hz}$ generated from 0 to $20 \mathrm{kHz}$, the minima, maxima, standard deviation, and mean have been selected as features. Additionally, the two global maxima and its position have been used. With these features, the correlations have been calculated to the target value of the height for the six inner steps of the reference object. An evaluation has been done with a heatmap. The highest correlation has been calculated with the $1,000 \mathrm{~Hz}$ windows between 6 and $18 \mathrm{kHz}$ using the local maxima. All achieved a negative correlation, this means, the higher the step of the reference object, the lower the value of the maximum in these windows. This effect can also be observed in figure 4. In the next step, these features have been used to train the ML model. For this purpose, the data set of 48 files, 8 per step, has been divided into $80 \%$ for training and $20 \%$ for testing sets. Different linear and polynomial regression models have been tested with the features described above. The best test results have been achieved with a polynomial degree of 2 . This model has been a coefficient of determination of 0.99 , a maximum deviation of the real value of all steps of $0.03683 \mathrm{~mm}$ and a MSE of 0.00036 $\mathrm{mm}$.

The evaluation of this model has been done by the determination of the height for each of the six steps of the reference object. Table 5 depicts the predicted values of the best model with the real value of the steps of the reference object and its difference. 
Tab. 5: Comparison of the test data set between the best model prediction for height and the real value

\begin{tabular}{cccc}
\hline step & real value & $\begin{array}{c}\text { predicted } \\
\text { value }\end{array}$ & deviation \\
\hline 2 & $27.03 \mathrm{~mm}$ & $27.05598 \mathrm{~mm}$ & $0.02598 \mathrm{~mm}$ \\
3 & $27.53 \mathrm{~mm}$ & $27.52823 \mathrm{~mm}$ & $-0.00177 \mathrm{~mm}$ \\
4 & $28.03 \mathrm{~mm}$ & $27.99317 \mathrm{~mm}$ & $-0.03683 \mathrm{~mm}$ \\
5 & $28.53 \mathrm{~mm}$ & $28.53649 \mathrm{~mm}$ & $0.00649 \mathrm{~mm}$ \\
6 & $29.01 \mathrm{~mm}$ & $29.01915 \mathrm{~mm}$ & $0.00915 \mathrm{~mm}$ \\
7 & $29.49 \mathrm{~mm}$ & $29.49254 \mathrm{~mm}$ & $-0.00254 \mathrm{~mm}$ \\
\hline
\end{tabular}

\subsection{Testing the principle with cutting inserts}

To test the potential of the new approach for TCM, three cutting inserts with the wear marks (VB $\left.B_{\max }\right) 0 \mu \mathrm{m}, 82 \mu \mathrm{m}$ and $90 \mu \mathrm{m}$ have been subjected to the same procedure. The inserts have been mounted in a tool holder and clamped in the machine vise in the machining centre. The nozzle has been vertically above the tool. According to figure 7a the nozzle has been moved across the inserts with the air jet to generate the specific impingement sound for each tool state. Beginning from the nose of the inserts with an offset of $0.2 \mathrm{~mm}$ and a path length of $25 \mathrm{~mm}$ the air jet has been crossing the tools three times. In total 21 sections have been recorded. This procedure is necessary, because the exact point of wear in relation to the air jet is not known. An example of the wear mark of an insert is depicted in figure $7 b$.
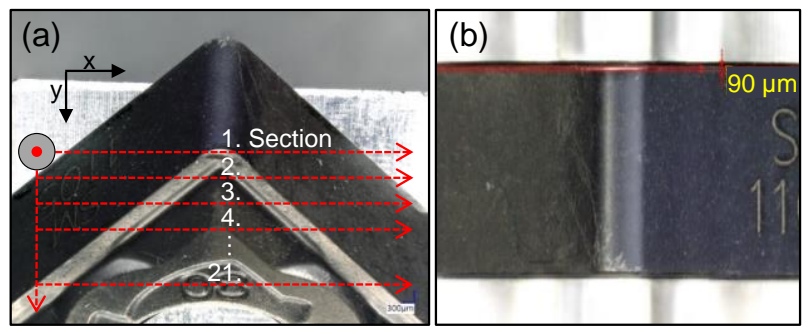

Fig. 7: Scheme of the nozzle movement for the cutting inserts (a), Example of an insert with $\mathrm{VB}_{\max }$ of $90 \mu$ (b)

According to section 3.5 the highest local maxima within $1,000 \mathrm{~Hz}$ windows between 6 to $18 \mathrm{kHz}$ have been applied as features to the 21 sections of the three inserts.

The dataset has been divided into $80 \%$ training data and $20 \%$ for testing. All 21 sections have been investigated. The best correlation has been achieved with section 19 for the inserts. To train the regression model the three inserts have been used and tested. With a polynomial degree of 2 the best model achieved an MSE of $12.358 \mu \mathrm{m}$ and a maximum deviation of the real value of $4.835 \mu \mathrm{m}$ across the three inserts. The result of the test data set is presented in table 6.

Tab. 6: Comparison of the test data set between the best model predictions for the wear marks $\left(V B_{\max }\right)$ and their real value

\begin{tabular}{ccc}
\hline real value & predicted value & deviation \\
\hline $0 \mu \mathrm{m}$ & $2.320 \mu \mathrm{m}$ & $2.320 \mu \mathrm{m}$ \\
$82 \mu \mathrm{m}$ & $77.165 \mu \mathrm{m}$ & $-4.835 \mu \mathrm{m}$ \\
$90 \mu \mathrm{m}$ & $85.542 \mu \mathrm{m}$ & $4.458 \mu \mathrm{m}$ \\
\hline
\end{tabular}

\section{DISCUSSION}

Due to the complexity of aeroacoustics effects and flow field characteristics, the functionality of the new approach has been depicted using a data-driven procedure. From simple shapes to its test on the tool wear of inserts different experiments have been applied. The results show the potential of measuring the height and width of an object as well as the tool wear of an insert. The accuracy and robustness of the new approach still need to be compared with the state-of-the-art in direct and indirect methods of measurement. The tool wear that is relevant for production is typically in a range between greater than 0 and $200 \mu \mathrm{m}$. With the new approach, distinctions of tool wear can be made in this range. The measurement accuracy and robustness for the application range must be further researched and improved. The experiments with the reference object have demonstrated that is possible to determine the height and width in a deviation between $0.00177 \mathrm{~mm}$ (minimum) and $0.03683 \mathrm{~mm}$ (maximum) to the real values. The deviations for measuring the width have been higher. The reason can be supposed by the different clamping because between the steps and the vise there are gaps, which could have an influence to the air stream.

In both investigations, the two outer steps have different effects according to the six inner steps referring to their spectrum (Fig. 4 and 5). These effects could be caused by the shapes of the outer steps, the air jet is only deflected on one side.

The adjustment of the setup has been done qualitatively. To achieve better measurement results for all potential influencing factors there should a quantitative assessment. Especially the nozzle and microphone need further investigations to find the best types. The tests have been carried out in the audible range; a test in the ultrasonic range could be advantageous. Directional microphones can also be useful to shield ambient noise.

The spectrums of the impingement sound present a broad sound spectrum with partial peaks. For a more appropriate and robust measurement approach, the root cause of the peaks and the patterns in the spectrums have to be more understood.

Three nozzles, two with a round flow and one with a flat flow have been used with two different settings of the air pressure ( 3 bar and 7 bar). The signals for the three nozzles were in a similar range. Further tests must be carried out with different nozzle types and air pressure settings to specifically influence the impingement sound as well as reduce process variations in the sound signals. This supports the further development of the new approach into a robust and accurate measurement process.

The feed rate of $500 \mathrm{~mm} / \mathrm{min}$ achieved better results than $1,500 \mathrm{~mm} / \mathrm{min}$. The reason for this could be that the slower the speed, the more measurement points are recorded for the same measurement time. The effects of slower feed rates still need to be tested. The functionality of the approach has been validated with three different inserts of the same shape. The deviation of the predicted measurand and the real value was in a range between $2.320 \mu \mathrm{m}$ and $4.835 \mu \mathrm{m}$, demonstrating the potential of using the new approach for TCM and measuring tool wear in grades. The sample size of the three inserts is very small. Further tests with a larger sample size and more increments still need to be performed.

With different measurands of height and width, the functionality of the new approach has been tested. Further gradations of the measurand and more complex objects should be tested. For the regression models, the features 
have been selected manually, it is expected that with automatic feature selection processes the accuracy of the models can be improved. Further, the generalization of the model still needs to be tested for the unlearned measurands.

\section{CONCLUSION}

This paper presents a new approach for TCM and object measurement, in which an air jet is directed over an object and the resulting impingement sound is used by ML models to infer its dimension and condition. The proof of concept has been demonstrated first with the application of simple shapes to measure their height and width. Second, the validation has been done by the tool wear of inserts. This new measuring approach can be an alternative to the existing TCM methods. For practical use of the new system, it must be further developed and compared with current TCM methods in terms of robustness and accuracy.

Benefits can be seen by using compressed air, which is available in almost all manufacturing areas. Thereby the new approach can easily be implemented. Additional application areas can be created with monitoring tasks based on the airborne sound with the microphone as part of the system.

This approach offers the potential to provide existing applications that use fluid jets, gases and liquids for tasks

\section{REFERENCES}

[Aliustaoglu 2009] Aliustaoglu, C., Ertunc, H. and Ocak, H. Tool wear condition monitoring using a sensor fusion model based on fuzzy inference system. Mechanical Systems and Signal Processing, 2009, pp. 539-546. ISSN 08883270.

[Alzahrani 2018] Alzahrani, A.M., Liu, R. and Kolodziej, J.R. Acoustic Assessment of an End Mill for Analysis of Tool Wear. Annual Conference of the PHM Society, 2018, Vol. 10, No. 1. ISSN 2325-0178.

[Bhattacharyya 2007] Bhattacharyya, P., Sengupta, D. and Mukhopadhyay, S. Cutting force-based real-time estimation of tool wear in face milling using a combination of signal processing techniques. Mechanical Systems and Signal Processing, 2007, pp. 2665-2683. ISSN 08883270.

[Bhuiyan 2014] Review of Sensor Applications in Tool Condition Monitoring in Machining. In: . Comprehensive Materials Processing. Elsevier. 2014, pp. 539-569. ISBN 9780080965338.

[Dimla 2000] Dimla, D.E. and Lister, P.M. On-line metal cutting tool condition monitoring. International Journal of Machine Tools and Manufacture, 2000, Vol. 40, No. 5, pp. 739-768. ISSN 08906955.

[Hwang 2019] Hwang. Bat-G net: Bat-inspired HighResolution 3D Image Reconstruction using Ultrasonic Echoes. In: H. Wallach; H. Larochelle; A. Beygelzimer; F. dltextquotesingle Alché-Buc; E. Fox; R. Garnett, eds. Advances in Neural Information Processing Systems. Curran Associates, Inc. 2019

[Kuntoglu 2020] Kuntoglu, M., Aslan, A., Sağlam, H., Pimenov, D.Y., Giasin, K. and Mikolajczyk, T. Optimization and Analysis of Surface Roughness, Flank Wear and 5 Different Sensorial Data via Tool Condition Monitoring System in Turning of AISI 5140. Sensors (Basel, Switzerland), 2020, Vol. 20, No. 16. such as cleaning, drying, ionizing, sorting and cooling with a monitoring and measurement function. A particular benefit is seen in processes where the process result is often controlled in a separate subsequent step, such as sandblasting and dry ice blasting.

\section{ACKNOWLEDGMENTS}

This article is part of the work of the Nuremberg Campus of Technology, a research cooperation between the University of Erlangen-Nuremberg and the University of Applied Sciences Nuremberg.

[Liu 2019] Liu, M.-K., Tseng, Y.-H. and Tran, M.-Q. Tool wear monitoring and prediction based on sound signal. The International Journal of Advanced Manufacturing Technology, 2019, Vol. 103, 912 , pp. 3361-3373. ISSN 0268-3768.

[Muehlbauer 2020] Muehlbauer, M., Würschinger, H., Polzer, D., Ju, S. and Hanenkamp, N. Automated Data Labeling and Anomaly Detection Using Airborne Sound Analysis. Procedia CIRP, 2020, Vol. 93, pp. 1247-1252. ISSN 22128271.

[Nguyen 2019] Nguyen, D.T., Maher, B. and Hassan, Y. Effects of Nozzle Pressure Ratio and Nozzle-toPlate Distance to Flowfield Characteristics of an Under-Expanded Jet Impinging on a Flat Surface. Aerospace, 2019, Vol. 6, No. 1, p. 4.

[Ostasevicius 2020] Ostasevicius, V., Karpavicius, P., Jurenas, V., Cepenas, M., Cesnavicius, R. and Eidukynas, D. Development of universal wireless sensor node for tool condition monitoring in milling. The International Journal of Advanced Manufacturing Technology, 2020, Vol. 110, 3-4, pp. 1015-1025. ISSN 0268-3768.

[Puente 2019] Puente León. Messtechnik. Berlin, Heidelberg: Springer Berlin Heidelberg, 2019. ISBN 978-3-662-59766-8

[Rehorn 2005] Rehorn, A.G., Jiang, J. and Orban, P.E. State-of-the-art methods and results in tool condition monitoring: a review. The International Journal of Advanced Manufacturing Technology, 2005, Vol. 26, 7-8, pp.693-710. ISSN 02683768 .

[Siddhpura 2013] Siddhpura, A. and Paurobally, R. A review of flank wear prediction methods for tool condition monitoring in a turning process. The International Journal of Advanced Manufacturing Technology, 2013, Vol. 65, 1-4, pp. 371-393. ISSN 0268-3768.

[Silva 1998] Silva, R.G., Reuben, R.L., Baker, K.J. and Wilcox, S.J. Tool wear monitoring of turning operations by neural network and expert system 
classification of a feature set generated from multiple sensors. Mechanical Systems and Signal Processing, 1998, Vol. 12, No. 2, pp. 319-332. ISSN 08883270.

[Vetrichelvan 2015] Vetrichelvan, G., Sundaram, S., Kumaran, S.S. and Velmurugan, P. An investigation of tool wear using acoustic emission and genetic algorithm. Journal of Vibration and Control, 2015, Vol. 21, No. 15, pp. 3061-3066. ISSN 1077-5463.

[Zhou 2018] Zhou, Y. and Xue, W. Review of tool condition monitoring methods in milling processes. The International Journal of Advanced Manufacturing Technology, 2018, Vol. 96, 5-8, pp. 2509-2523. ISSN 0268-3768. 